audiotaping. For example, one of our best teaching fellows discovered she had idiosyncratic habitual motions that she had been entirely unaware of. Another was very surprised to see the expressions on the students' faces when he was writing on the blackboard with his back to the class.

H. Shipman: I agree; videotaping should be done if available. Audio tapes are better than nothing.

\title{
TEACHING 7000 STUDENTS PER YEAR
}

\section{R. Robert Robbins,}

Department of Astronomy, University of Texas, Austin, Texas 78712, U.S.A.

The undergraduate program at the University of Texas has grown into the largest astronomy teaching program in the world, with some 7000 students per year (almost 20,000 credit hours). The department has 22.5 Ph.D.-level teaching faculty, about 45 graduate students, and about 40 pre-professional undergraduate majors. But most of the enrollment is in courses that satisfy the science requirements of students in liberal arts and non-technical majors. In 1985-86, 96.4 per cent of our undergraduate credit hours taught were in such classes. It is instructive to examine the historical reasons for our growth and its educational consequences, and to draw some conclusions from both for other programs.

\section{The Early Growth of the Department: "Reclaiming" the McDonald Observatory}

Up until 1958, there was no astronomy department at all at the University of Texas - only the McDonald Observatory, named for the Texas banker who left the money to build it in 1926. It was run by Yerkes Observatory, but when efforts began to bring the University of Texas into the first rank of academic institutions worldwide, it was decided to develop a competitive, major astronomy department in Austin and "reclaim" the observatory from the University of Chicago. To this end, in 1963, Dr. Harlan Smith was brought in to be both the director of the observatory and the chairman of the new department; shortly thereafter, the 2.7-m (107") telescope was begun at the observatory. (For more details, see Big and Bright: A History of the McDonald Observatory, by David Evans and Derral Mulholland (University of Texas Press, 1986)).

The original goals called for the new department to grow to about 12 faculty members, a number judged appropriate to support a major observatory. However, it has now grown to almost double this targeted size, and we will see below that undergraduate teaching has been responsible for this. 
The "Post-Sputnik" Degree Plan at the University and the Influence of the "Space Age"

The figure shows that student enrollments did not begin to increase significantly until the school adopted a new degree plan in 1967, requiring 15 hours of science in every major at the University. This is one of the strongest science requirements in the country, and it immediately began to generate growing enrollments in the service courses of all the science departments. Astronomy became the science department that was most successful in acquiring new enrollments, no doubt partially aided by the well-publicized space program of the United States in the 1960's and the 1970's, which directed the attention of much of the world towards astronomy. (Note: the University grew by about 50 per cent over this period, not enough to explain our huge growth. And grade statistics show that the enrollments cannot be attributed to easy grading.)

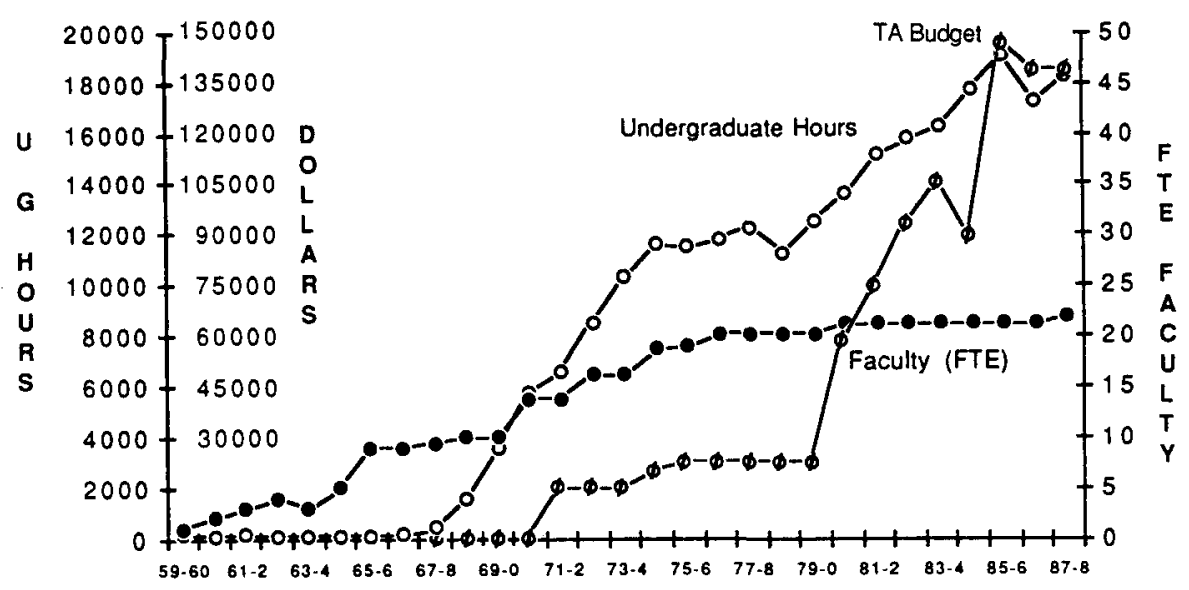

Fig. 1. Credit hours, teaching assistant budget, and number of faculty in the astronomy department at the University of Texas.

\section{"Intangible" Factors Creating Sustained (i.e., Subsequent, Continuing) Growth}

It must be noted that many other large universities also had the potential to use the widespread interest in astronomy to build up sizable departments, but in reality it did not happen very often. To explain our continued growth over two decades, I feel that it is necessary to turn to an intangible factor.

Many astronomy departments concentrate their resources almost exclusively at the graduate level. At the University of Texas, it was decided early on to also put significant effort and funding into undergraduate programs for non-science majors. It began with a "critical mass" of the early faculty members who were interested enough in undergraduate education to influence the subsequent development of the 
department. In the late 1960's, this group included Dr. Smith and me, and also Frank Bash and Bill Jefferys. An overall commitment of the whole department did not occur all at once, but developed over time. But one can isolate some of the positive factors that grew from this departmental commitment:

1. All the faculty teach classes for non-science majors at least once a year. All the faculty take the needs of the program seriously, since they are all involved in it.

2. The first class in astronomy (for non-science majors) is a very accessible, general non-mathematical survey of the universe, covering the contents of the universe and the astrophysical models that modern astronomy constructs from its observations.

The lack of math is simply an unfortunate necessity, because American college students are typically not prepared to handle any level of mathematics at all. We have decided that we cannot right all the wrongs of the American secondary school system in one course, but we can give the students a powerful contact with the modern universe, an exposure that in many cases may significantly affect their lives. The lack of math does not necessarily imply a substandard course, because the high degree of abstract thinking and reasoning skills that are demanded by the sophisticated syllabus of modern astronomy guarantees that the course will be one of the more challenging in the undergraduate program (See Robbins, Annals of Engineering Education, Dec. 1981, p. 208, for a study of the factors that determine student success in introductory astronomy).

The introductory course is presented in three modes: (i) A conventional lecture with an optional 1-unit lab. However only about 5 per cent of the students elect the lab. (ii) A lecture class with observing activities blended into it, that is, it has a lab component integrated into the big class itself, and (iii) a large self-paced (no lectures, Keller-method) reading class taken by about 900 students per year. These options provide variety for students with different types of study skills.

The first class is then followed by an attractive smorgasbord of followup classes. About 70 per cent of our introductory students go on to a second semester of astronomy, so this approach is obviously successful. These classes are also nonmathematical, but they develop a particular area of astronomy in more depth. Some examples are: Man in the Solar System; The Search for Extraterrestrial Life; Stars and Stellar Evolution; Astronomy in Science Fiction; Galaxies, Quasars, and Cosmology, The Milky Way Galaxy; Archaeo- and Ethno-Astronomy of the Americas; and History and Philosophy of Astronomy. These courses are designed by the various faculty in their areas of greatest interest, and this freedom seems to result in follow-up classes taught with enthusiasm.

3. We have consistently given time and money to a vigorous outreach program that produces brochures, film series and star parties, and other programs of high visibility, and funds an educational services office with a staff of 3 people to 
handle public relations and assist with the audiovisual and other needs of large classes.

A central lesson to be drawn from our Texas experience is that to develop a significant undergraduate enrollment, the decision must be consciously taken that undergraduate education is important and should be encouraged and supported. It doesn't happen by itself.

The graph demonstrates that our growing enrollment did create a momentum that carried the department past its targeted size of 12 faculty members to almost twice as large. But it also shows that since the mid-1970's, increased enrollment has not generated new faculty positions.

\section{Our "Mature" Department and Its Natural Enrollment Limit}

Today, most of the students who are allowed to take astronomy by their degree programs do so. We are quite close to the maximum possible enrollment. But we are also up against another limit, which is (1) the number of classes we teach times (2) the number of students that will fit into our largest lecture hall (230). The only way to expand further would be to acquire more faculty or to seek out larger classrooms. I do not know of any research that settles the question of what is the optimum size for a class, but by anyone's standards, ours are all quite large already!

Does the increased enrollment inevitably result in increased budget and faculty lines? The Texas experience suggest "Yes, but..." since there may be a considerable time lag (i.e., years or decades; see the graph) before the support appears. There will typically be no immediate reward for "overteaching."

\section{Some Educational "Pros" and "Cons" of Our Size}

An advantage of large programs is that they have a great diversity of offerings to select from, to appeal to the wide range of interests that students can exhibit. But many of the drawbacks to a very large program are fairly obvious to any experienced educator.

1) Evaluation and Grading: Evaluating huge classes can become very timeconsuming. Most instructors of very large classes eventually turn to multiple-choice exams. I do not intend to present a point of view on what form of testing is superior; I am simply pointing out consequences.

2) Labs and Observations: Although a "lab" experience is probably the best way to teach astronomy, at Texas fewer than 5 per cent of the students get one. This is the principal source of disappointment noted on student evaluations. It is simply not possible to offer an intensive laboratory class for as many students as we have; we do not have the rooms, equipment, or instructors. One lecture section does offer measurement experiences to its students, but the instructor teaching that class must replace conventional homework assignments with observing activities, and many instructors prefer not to do this.

3) Less personal contact with the instructor: Students will most commonly 
interact personally with a Teaching Assistant. The graph shows that funding for TA positions is the only factor that has tried to keep pace with the growing enrollments. We generally employ about 20 TA's per semester, at 20 hours per week (half-time), which still only results in about 1.5 TA's for each class of 230 students.

A considerable problem for the teaching program is that the research programs at Texas will often hire most of the astronomy graduate students as Research Assistants. Thus, we must hire some of our TA's from other departments (physics, engineering), and to try to train them for the job. Clearly, such TA's will be much more limited in the duties they can carry out. Also, many of the astronomy TA's today are foreign students who often have such a poor command of the English language that they are quite restricted in their usefulness.

\title{
Summary
}

Whether or not you feel that the Texas experience is directly or only partially applicable to your institution, it does indicate the ultimate potential of an undergraduate program. If all U.S. colleges would develop programs comparable to ours, the job market for astronomy teachers would increase by at least a factor of 10 ! Imagine the effect of that on astronomy as a career.

In researching this paper, I personally was surprised by the importance of intangible factors, and most particularly, the importance of the belief that undergraduate education is important and worthy of effort. This factor explains why growth was allowed to continue, even when the tangible rewards from it were not always evident.

\section{WORKSHOP FOR TEACHERS OF INTRODUCTORY ASTRONOMY}

\author{
George S. Mumford \\ Department of Physics and Astronomy, Robinson Hall, Tufts University, Medford, \\ Massachusetts 02155 U.S.A.
}

As interest in astronomy develops through missions to Mars, SETI, and heavenonly-knows-what earth-shaking new discoveries in the future, demand for astronomy courses at all levels will increase. Without adequate numbers of professional astronomers to teach them, persons from other fields will be thrown into the breech. Already a significant number of college students in the United States are receiving instruction from persons not trained in astronomy. I suspect that this is currently true world-wide, especially as physicists who adopt our field for their research on neutrinos or cosmic strings are assigned or volunteer to teach elementary courses. 